\title{
Predictors of Health-Related Quality of Life and Communicative Participation in Individuals with Dysarthria from Parkinson's Disease
}

\author{
Kristie A Spencer*, Clare Friedlander and Katherine A Brown \\ University of Washington, USA

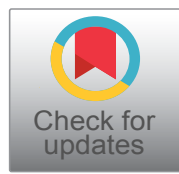

*Corresponding author: Kristie A Spencer, Ph.D, Professor, Associate Chair, University of Washington, 1417 NE $42^{\text {nd }}$ St., Seattle, WA 98105, USA, Tel: 206-543-7980

\begin{abstract}
Purpose: The motor and non-motor deficits of Parkinson's disease (PD) can cause daily challenges and have been associated with reduced health-related quality of life ( $\mathrm{HrQoL})$. A number of variables have been consistently, though not unequivocally, identified as important in influencing overall HrQoL in individuals with PD, such as demographic factors, cognitive decline, and level of motor impairment. However, the presence and severity of dysarthria is often overlooked, despite the potentially adverse influence on HrQoL. The primary purpose of this study was to understand the predictors of $\mathrm{HrQoL}$, as well as self-perceived communication participation, in individuals with PD who have dysarthria.

Methods: Twenty-seven participants with Parkinson's disease and dysarthria completed the study. Linear regression was used to determine the predictive ability of motor function, speech intelligibility and cognitive performance on HrQoL and communicative participation; models were run with and without the demographic/clinical variables of age, sex, years of education, disease duration, and depression scores.

Results: HrQoL was significantly predicted by the severity of motor symptoms and the combination of cognitive functioning with age, whereas communicative participation was only predicted by monologue intelligibility.

Conclusions: This preliminary study suggests that the HrQoL of individuals with dysarthria from PD may be most affected by overall motor function, while participation in life situations may be most influenced by decreased conversational intelligibility.
\end{abstract}

\section{Introduction}

Parkinson's Disease (PD) affects more than 8 million people globally [1] and is considered a complex multidimensional disorder that leads to a broad array of motor and non-motor symptoms [2]. The primary motor characteristics of PD include tremor, rigidity, bradykinesia/akinesia, and postural instability; secondary motor characteristics include dysarthria, dysphagia, and micrographia. Non-motor symptoms emerge from numerous domains, including cognitive, neuropsychiatric, sleep, sensory, and autonomic systems [3], and are present in the majority of people with PD.

Both the motor deficits $[4,5]$ and non-motor deficits [6-8] can cause daily challenges and have been associated with reduced quality of life. The motor impairments often lead to decreased physical mobility, which is the most commonly reported problem among people with PD $[4,5]$. Additionally, motor impairments negatively impact the ability to complete activities of daily living (ADLs) which can lead to a decreased level of independence. Non-motor deficits, particularly cognitive impairment, are commonly observed across the course of PD, with approximately $15 \%-25 \%$ of people presenting with challenges in the early stages of the disease and $50-80 \%$ of people demonstrating dementia over the course of the disease $[9,10]$. The characteristics of cognitive impairment vary by person, but may include a decline in executive function, attention, memory, working memory, and visuospatial functioning [10-13]. Cognitive deficits in individuals with PD have been associated with decreased independence, decreased participation in communication exchanges, and increased levels of de-

Citation: Spencer KA, Friedlander C, Brown KA (2020) Predictors of Health-Related Quality of Life and Communicative Participation in Individuals with Dysarthria from Parkinson's Disease. Int J Neurodegener Dis 3:014. doi.org/10.23937/2643-4539/1710014

Accepted: January 01, 2020; Published: January 03, 2020

Copyright: (c) 2020 Spencer KA, et al. This is an open-access article distributed under the terms of the Creative Commons Attribution License, which permits unrestricted use, distribution, and reproduction in any medium, provided the original author and source are credited. 
pression [14]. Deficits in executive functions, in particular, have been linked to increased difficulty completing instrumental ADLs, such as driving, cooking a meal, effectively administering medications, and handling personal finances [15-17].

Measures of health-related quality of life (HrQoL) have frequently been used to capture areas of functioning that can be affected when living with chronic illness [18]. HrQoL is considered a multi-dimensional metric that emphasizes the impact of health status, and includes various domains, such as physical, mental, emotional, and social health. A number of variables have been consistently - although not unequivocally - identified as important in influencing overall HrQoL in individuals with PD [19]. These variables most often include demographic characteristics such as age $[20,21]$, stage of illness [22], movement symptom severity and type $[5,23]$, and decreased cognitive function $[10,17,24]$.

In studies of HrQoL, participants with PD are often well characterized in terms of general motor and cognitive function; however, the presence/severity of dysarthria is often overlooked. As dysarthria occurs in the majority of people with PD (70-90\%; Sapir, 2014) [25], and can lead to significant functional limitations [26], its inclusion in studies of HrQoL is warranted. Hypokinetic dysarthria from PD can cause impairments across respiration, phonation, articulation and prosody. Dysarthria is regarded as the greatest challenge facing $10-30 \%$ of people with PD $[27,28]$. These speech changes can lead to decreased motivation to communicate [14] as well as decreased conveyance of emotions via speech [29]. Additionally, hypokinetic dysarthria frequently results in decreased speech intelligibility. More than $50 \%$ of people with PD will present with reduced speech intelligibility over the course of the disease [28]; this change to intelligibility has been shown to negatively impact communication participation [30-32]. Moreover, unlike the other motor deficits of PD, dysarthria is more apt to lead to changed relationships and a loss of connection with family and friends [33].

The primary purpose of this preliminary study was to understand the predictors of HrQoL in individuals with PD who have dysarthria. The determinant ability of four sets of predictor variables were considered: Demographic/clinical variables, motor functioning, cognitive ability, and speech impairment. Given the presence of dysarthria in the participants, we were additionally interested in whether the same predictor variables would influence communication participation as a secondary goal. Communicative participation is defined as "taking part in life situations in which knowledge, information, ideas or feelings are exchanged" $[34,35]$. It reflects how a condition, such as PD, interferes with a wide range of daily communication activities [34]. At present, the determinant ability of the aforementioned predictor variables on communication participation is largely un- known (but see McAuliffe, et al. [30] for a study using self-report measures).

In sum, a number of variables have been consistently identified as having an important influence on HrQoL in individuals with PD, including demographic/clinical factors, motor symptom severity, and decreased cognitive function. However, the prevalent and impactful variable of dysarthria has yet to be considered in studies of HrQoL in PD. Thus, this study aims to examine the determinant ability of four sets of variables-demographic/clinical, motor impairment, cognitive functioning, and speech impairment-on HrQoL and communication participation in individuals with PD. The relative influence of dysarthria is important to understand when educating people with $\mathrm{PD}$ and being proactive in terms of management, that is, anticipating disease sequelae that are more likely to lead to decreased quality of life or decreased communicative participation. It was predicted that speech intelligibility would be a strong predictor of both HrQoL and communication participation in individuals with PD and dysarthria.

\section{Methods}

The study was approved by the Institutional Review Board at the University of Washington. Following informed consent, participants completed background demographic and disease-related questions.

\section{Participants}

Inclusion criteria were (1) Diagnosis of idiopathic PD by neurologist (per self-report), (2) Native speaker of American English, (3) Typical speech, language, and cognitive developmental history, and (4) Presence of dysarthria based on independent determination of perceptible characteristics of speech disruption by two investigators. Exclusion criteria included (1) History of Deep Brain Stimulation, (2) Diagnosis of secondary Parkinsonism, (3) Severe depression per Beck Depression Inventory II (BDI-II) score $\geq 29$, (4) Neurologic compromise beyond PD (e.g., stroke, traumatic brain injury), (5) Alcohol or drug dependency, (6) Currently taking sedatives or tranquilizers, and (7) Vision or hearing inadequate to permit participation in the study.

A total of 27 individuals participated in this study. The age of participants ranged from 60 to 81 years $($ mean $=71.11, S D=5.78)$, with more males $(n=18)$ than females. Duration of disease varied from 1 year to 20 years (mean $=9.06, S D=4.82$ ) and education ranged from 13 to 22 years (mean $=17.07, S D=1.92$ ).

\section{Procedure}

Participants completed an assessment of motor, cognitive and speech function, as well as a measure of HrQoL and communication participation.

Motor functioning: Following guidelines of The In- 
ternational Parkinson and Movement Disorder Society (MDS), two researchers were trained in the use of the MDS-Unified Parkinson's Disease Rating Scale (UPDRS), Motor Examination (Part III; Goetz, et al.) [36]. Motor symptoms were characterized via 13 Motor Examination scores and 3 self-report scores from Part II, Motor Aspects of Experiences of Daily Living. Total motor score was calculated as the mean of the following 16 motor sections. Overall motor functioning was also assessed using the modified Hoehn and Yahr scale [37].

Speech functioning: Speech was recorded using a high-quality, head-mounted microphone (AKG C520) with a constant mouth-to-microphone distance of two inches. The microphone was connected to a portable digital speech recorder (Zoom H6, GU-ZOOMH6). All speech samples were recorded in a quiet environment with low ambient noise.

Two tasks were used to elicit speech samples. For a quantifiable index of each speaker's sentence-level intelligibility, the computerized version of the Speech Intelligibility Test (SIT; Yorkston, Beukelman, \& Hakel) [38] was administered. This task included 11 randomized, computer-generated sentences of 5 to 15 words in length. The SIT was scored following the Yorkston, et al. [38] protocol. In addition, a monologue was elicited during a description task, where participants were asked to talk about their job, their family or a memorable vacation for approximately 60 seconds. Samples were segmented into speech runs, which were operationally defined as a stretch of speech bounded by a silent period or pause between words of at least 200 milliseconds [39]. Each monologue was transcribed by a researcher to establish the 100-word speech run according to standard transcription criteria [8,39-41]. For reliability purposes, an independent judge reassessed $15 \%$ of the monologues, to ensure criteria for speech runs were applied consistently with the initial coding.

The intelligibility of the 100-word speech samples from each personal narrative was subsequently scored via transcription by three independent listeners who were native English speakers without hearing loss. All speech samples were transcribed in a quiet environment with low ambient noise. Transcription accuracy was averaged across the listeners for a mean monologue intelligibility scores for each speaker with PD.

Cognitive functioning: The Montreal Cognitive Assessment (MoCA; Nasreddine, et al.) [42] was chosen as it has shown good sensitivity in identifying the specific cognitive deficits in PD $[9,43,44]$. The following MoCA scores align with established cognitive profiles: Cognitive impairment (overall score of $\leq 21$ ), mild cognitive impairment (overall score of $<26$ ), and normal cognitive function (overall score $\geq 26$ ) [42].

Health related quality of life and communication participation: HrQoL was measured using the Parkin- son's Disease Questionnaire-8 (PDQ-8), which is a disease-specific measure used to identify an individual's overall health-related quality of life (HrQoL; Jenkinson, et al.) [45]. The PDQ- 8 was developed as a shorter version of the Parkinson's Disease Questionnaire-39 (PDQ39; Jenkinson, Fitzpatrick, Peto, Greenhall, \& Hyman) [46]. Each of the eight questions on the PDQ-8 correlate with an overall domain represented on the PDQ-39 (e.g., mobility, cognition), and the short-form has been confirmed as a reliable and valid measure of $\mathrm{HrQoL}$ in PD $[45,47]$. The Communicative Participation Item Bank (CPIB) was used to describe the interference of PD with an individual's communication participation [34]. The CPIB evaluates how a condition, such as PD, interferes with a wide range of daily speech communication activities, using a four point scale [34]. Participants were asked to consider how changes to their speech affected their day-to-day participation in communicative situations.

Statistical analyses: Single and multiple linear regression models were used to determine the role of motor function, speech intelligibility and cognitive performance as predictors of HrQoL and communicative participation. Regression models were run with and without the demographic/clinical variables of age, sex, years of education, disease duration, and depression scores per the BDI-II. An alpha level of 0.05 was used. Correlations (Pearsons $r$ : two-tailed) were used to assess the degree of relationship between each potential determinant -demographic/clinical, cognitive, motor and speech variables with health-related HrQoL and communication participation.

\section{Results}

\section{Reliability}

Inter-rater reliability for identifying speech runs for $15 \%$ of the monologues was determined to be $97.8 \%$.

\section{Motor, speech, and cognitive measures}

Measures of motor function, speech intelligibility and cognitive abilities, averaged across participants, are shown in Table 1. There was a broad range of motor severity as measured by the UPDRS and Hoehn and Yahr scales [48], with participants ranging from mild, unilateral motor signs to marked, bilateral motor signs. Average speech intelligibility was high for both the SIT (mean 96\% transcription accuracy) and monologue (mean 94\% transcription accuracy). Cognitive function, as measured by the MoCA, was distributed across severity levels, ranging from normal functioning (score of 30 ) to cognitive impairment (score of 16). Average performance fell in range of mild cognitive impairment.

\section{Predictors of Health Related Quality of Life (HrQoL)}

Linear regression analyses were conducted to in- 
Table 1: Measures of motor function, speech intelligibility and cognitive performance.

\begin{tabular}{|l|l|l|l|}
\hline Variables & Mean & SD & Range \\
\hline Motor Function & & & \\
\hline $\begin{array}{l}\text { UPDRS Score (max = 32) } \\
\text { Hoehn \& Yahr Scale (max = 5) }\end{array}$ & 12.89 & 6.99 & $3-32$ \\
\hline Speech Intelligibility & & 0.77 & $1-4$ \\
\hline SIT (\%) & 95.96 & 2.49 & $89.09-100$ \\
\hline Monologue (\%) & 93.59 & 4.78 & $81.33-100$ \\
\hline Cognitive Function & & & \\
\hline MoCA (max 30) & 24.70 & 4.18 & $16-30$ \\
\hline
\end{tabular}

Note: UPDRS: Unified Parkinson's Disease Rating Scale, higher score reflects more impairment; SIT: Sentence Intelligibility Test; MoCA: Montreal Cognitive Assessment, lower score reflects more impairment.

Table 2: Predictors of Health-Related Quality of Life per Parkinson's Disease Questionnaire-Short-Form (PDQ-8) based on Linear Regression Models.

\begin{tabular}{|l|l|l|}
\hline Motor Variables & p-value & Adjusted $\mathbf{R}^{\mathbf{2}}$ \\
\hline UPDRS + Clinical/Demographics & 0.068 & 0.241 \\
\hline H \& Y + Clinical/Demographics & 0.174 & 0.139 \\
\hline UPDRS & $0.001^{*}$ & 0.322 \\
\hline H \& Y & $0.014^{*}$ & 0.189 \\
\hline Speech Variables & $\mathbf{p}$-value & Adjusted R \\
\hline SIT + Clinical/Demographics & 0.480 & -0.011 \\
\hline Monologue + Clinical/Demographics & 0.390 & 0.025 \\
\hline SIT & 0.961 & -0.040 \\
\hline Monologue & 0.306 & 0.003 \\
\hline Cognitive Variable & $\mathbf{p}$-value & Adjusted $\mathbf{R}^{\mathbf{2}}$ \\
\hline MoCA + Clinical/Demographics & $0.043^{*}$ & 0.284 \\
\hline MoCA & 0.111 & 0.062 \\
\hline
\end{tabular}

" $\mathrm{p}<0.05$ Note: UPDRS: Unified Parkinson's Disease Rating Scale; H \& Y: Hoehn and Yahr scale; SIT: Speech Intelligibility Test, MoCA: Montreal Cognitive Assessment.

Table 3: Pearson correlation coefficients for predictor and response variables.

\begin{tabular}{|l|l|l|}
\hline & Response & Variables \\
\hline Predictor Variables & PDQ-8 & CPIB \\
\hline UPDRS & $-0.590^{\star *}$ & -0.338 \\
\hline H \&Y & $-0.469^{*}$ & -0.072 \\
\hline MoCA & -0.314 & -0.092 \\
\hline SIT & 0.010 & 0.056 \\
\hline Monologue & 0.205 & $0.609^{\star *}$ \\
\hline Age & -0.336 & -0.214 \\
\hline Education & -0.153 & -0.113 \\
\hline Disease Duration & -0.154 & -0.210 \\
\hline BDI-II & -0.123 & -0.146 \\
\hline
\end{tabular}

"correlation is significant at the 0.05 level (two-tailed) "correlation is significant at the 0.01 level (two-tailed) vestigate the relationship between HrQoL and motor functioning, speech functioning and cognitive functioning. Regression models were run with and without inclusion of the demographic/clinical variables of age, sex, years of education, disease duration, and depression scores (BDI-II).

Both measures of motor functioning, the UPDRS and Hoehn and Yahr scale, were found to be predictive of performance on the PDQ-8, but only when the demographic/clinical variables were not factored into the model. As shown in Table 2, the UPDRS was a significant predictor of $\mathrm{PDQ}-8$ performance $(\mathrm{F}(1.25)=$ $13.34, p<0.1$ ), with an $R^{2}$ of 0.32 .A significant regression equation was also found using the Hoehn and Yahr scale as a predictor of PDQ-8 $(F(1.25)=7.04, p$ $<0.5)$, with an $\mathrm{R}^{2}$ of 0.19 . A Pearson product-moment correlation coefficient was computed to assess the nature of the relationship between the PDQ- 8 and the two motor scales (Table 3 ). There was a significant negative correlation between performance on the PDQ- 8 and the UPDRS $(r=-0.590, p<0.1)$ as well as the Hoehn and Yahr scale $(r=-0.469, p<0.5)$. Thus, scores on the PDQ-8 suggesting worse HrQoL were significantly associated with more impaired motor presentation on both the UPDRS and Hoehn and Yahr measures.

Speech intelligibility as a predictor of HrQoL, with and without inclusion of demographic/clinical variables, is shown in Table 2. Linear regression analyses revealed no significant relationships between HrQoL and speech intelligibility measures. Pearson correlations between intelligibility measures and HrQoL were not significant (Table 3).

With the MoCA, a significant regression equation was found $(F(6.20)=2.72, p<0.5)$, with an adjusted $R^{2}$ of 0.28 . However, the MoCA was only a significant predictor of PDQ-8 performance when combined with the demographic/clinical variables (Table 2 ). In that model, both the MoCA $(p=0.09)$ and age $(p=0.04)$ uniquely accounted for variance in the PDQ-8. The MoCA by itself, without the demographic variables, was not a significant predictor $(p=0.11)$. Pearson correlations between the MoCA and HrQoL were not significant (Table 3).

\section{Predicting communication participation}

Linear regression analyses were conducted to investigate the relationship between communicative participation (per the CPIB) and motor functioning, speech functioning and cognitive functioning. Regression models were run with and without the demographic/clinical variables of age, sex, years of education, disease duration, and depression scores (BDI-II).

The role of motor characteristics as a predictor of communicative participation, with and without the demographic/clinical variables, is shown in Table 4. Linear regression analyses revealed no significant re- 
Table 4: Predictors of communicative participation per the Communicative Participation Item Bank (CPIB) based on Linear Regression Models.

\begin{tabular}{|l|l|l|}
\hline Motor Variables & p-value & Adjusted $\mathbf{R}^{2}$ \\
\hline UPDRS + Clinical/Demographics & 0.377 & 0.031 \\
\hline H \& Y + Clinical/Demographics & 0.475 & -0.009 \\
\hline UPDRS & 0.085 & 0.079 \\
\hline H \& Y & 0.720 & -0.035 \\
\hline Speech Variables & $p$-value & Adjusted R \\
\hline SIT \& Clinical Demographics & 0.441 & 0.005 \\
\hline Monologue \& Clinical Demographics & $0.010^{*}$ & 0.402 \\
\hline SIT & 0.782 & -0.040 \\
\hline Monologue & $0.001^{* *}$ & 0.346 \\
\hline Cognitive Variable & $p$-value & Adjusted $R^{2}$ \\
\hline MoCA \& Clinical Demographics & 0.450 & 0.001 \\
\hline MoCA & 0.647 & -0.032 \\
\hline
\end{tabular}

" $p<0.05$; " $p<0.01$ Note: UPDRS: Unified Parkinson's Disease Rating Scale; H \& Y: Hoehn and Yahr scale; SIT: Speech Intelligibility Test; MoCA: Montreal Cognitive Assessment.

lationships between motor performance measures and communication participation. Pearson correlations between motor measures and communication participation were not significant (Table 3).

Speech intelligibility as a predictor of communicative participation is shown in Table 4. Monologue intelligibility was found to be predictive of performance on the CPIB, both with $\left(F(1.25)=3.91, p<0.001 ; R^{2}=0.40\right)$ and without $\left(F(1.25)=14.73, p<0.001 ; R^{2}=0.35\right)$ inclusion of demographic/clinical variables. A Pearson product-moment correlation coefficient was computed to assess the nature of the relationship between the CPIB and the two measures of speech intelligibility (Table 3). There was a significant correlation between monologue intelligibility and the CPIB $(r=0.609, p<0.001)$. Thus, scores on the CPIB suggesting worse communicative participation were significantly associated with decreased monologue speech intelligibility. The correlation between the CPIB and SIT was not significant.

Cognition (measured by the MoCA) as a predictor of communicative participation, with and without the demographic/clinical variables, is shown in Table 4. Linear regression analyses revealed no determinant ability of communicative participation by the MoCA. Pearson correlations between the cognitive measure and communicative participation were not significant.

\section{Discussion}

The purpose of this preliminary study was to evaluate factors influencing HrQoL and, as a secondary goal, communicative participation in people with PD and dysarthria. Predictors reflecting the movement disorder domain, the cognitive domain, and general demographic/clinical variables were chosen based on research suggesting their relatively consistent influence on HrQoL.
However, the speech domain was also included as it has been largely neglected when considering predictors of HrQoL, despite the prevalence and challenge of dysarthria in people with PD. A measure of communicative participation was also included to expand the breadth of measures of self-perceived well-being, particularly given the presence of dysarthria in our sample. HrQoL was significantly predicted by the severity of motor symptoms and, to a lesser extent, cognitive functioning, whereas communicative participation was only predicted by monologue intelligibility.

Both movement disorder metrics were found to be predictors of HrQoL per the PDQ-8; speech intelligibility was not predictive of HrQoL despite the presence of dysarthria in the sample. The PDQ-8 measures HrQoL across eight domains of health and illness, including mobility, cognition, emotional well-being, communication and bodily discomfort. However, a relatively larger percentage of items are related to motor functioning (e.g., getting around in public, dressing, painful muscle cramps) and could have influenced the findings. That said, our results are consistent with a wealth of literature suggesting the strong predictive influence of motor functioning on overall $\mathrm{HrQoL}[4,5,23]$. Specifically, an elevated severity score on the UPDRS, consistent with more severe tremor, slowness, rigidity, etc., and higher scores on the Hoehn and Yahr scale, associated with more distributed and severe symptoms of PD, were associated with reduced HrQoL.

In addition to measures of motor functioning, the MoCA was found to be a significant predictor of HrQoL, but only when combined with the variable of age. However, the MoCA did not significantly predict performance on the PDQ- 8 when measured alone, indicating that a predictive relationship does not exist between the two without the influence of other factors. The MoCA is known to have pronounced age effects, particularly when considered with an individual's level of education $[49,50]$. Thus, increased age, when coupled with poorer cognitive performance on the MoCA, may be associated with worsened HrQoL in individuals with Parkinson's disease.

Findings from this study revealed several predictive relationships when considering determinants of communicative participation as measured by the CPIB. Monologue intelligibility was observed to significantly predict performance on the CPIB, both with and without the demographic/clinical variables. Motor severity measures, the SIT, and cognitive variables were not found to be predictive of communicative participation as measured by the CPIB. Thus, decreased conversational intelligibility negatively impacts communicative participation while the SIT had no relationship with the CPIB. The divergence between these two measures of intelligibility was unexpected. It is possible that the SIT inflated the participants' intelligibility, due to the improved un- 
derstandability and naturalness that often occurs when individuals with PD are externally cued by a reading task $[40,51]$. Therefore, clinicians (and researchers) should consider measurement of intelligibility via monologue whenever possible, as it appears to be the more sensitive clinical tool, particularly when attempting to anticipate impact to communication participation.

The predictive relationship between monologue intelligibility and communicative participation illustrates the pronounced impact of dysarthria on engagement in life activities. It is well documented that the communication changes associated with hypokinetic dysarthria cause individuals with PD to demonstrate a significant decrease in their self-perceived communication skills $[14,27,28]$, but the specific relationship between intelligibility and communicative participation has not been sufficiently studied [30,31,52].

It is perhaps surprising that the MoCA was not predictive of communication participation as cognition is foundational to communication. That said, the MoCA may not have been sufficiently sensitive or comprehensive to fully assess the cognitive abilities of persons in this study $[2,42]$. Previous research has noted that increasing the difficulty of certain items on the MoCA (e.g., orientation information) may increase its sensitivity for this population [2].

\section{Limitations and future directions}

These conclusions should be viewed as preliminary given the small sample size and subsequent reduced ability to generalize to the larger population of people with PD. By extension, our findings may have been influenced by the milder severity of dysarthria in our sample, given that no speakers fell below $81 \%$ intelligibility. It is possible that speech intelligibility would have had a stronger influence on HrQoL if a broader range of severity was included. Our sample was, however, more diverse in terms of overall motor functioning (UPDRS 3-32; Hoehn and Yahr 1-4) and cognitive functioning (MoCA 16-30). Overall, these results indicate a combination of speech and motor deficits may be the best predictor of overall quality of life. Improved awareness and understanding of the relationship between changes to communicative participation and HrQoL will encourage a more complete and comprehensive approach to patient care $[53,54]$.

\section{References}

1. Gopalakrishna A, Alexander SA (2015) Understanding parkinson disease: A complex and multifaceted illness. J Neurosci Nurs 47: 320-326.

2. Kletzel SL, Hernandez JM, Miskiel EF, Mallinson T, Pape TL (2017) Evaluating the performance of the montreal cognitive assessment in early stage parkinson's disease. Parkinsonism Relat Disord 37: 58-64.

3. Marras C, Chaudhuri KR (2016) Nonmotor features of parkinson's disease subtypes. Mov Disord 31: 1095-1105.
4. Karlsen KH, Larsen JP, Tandberg E, Maeland JG (1999) Influence of clinical and demographic variables on quality of life in patients with Parkinson's disease. J Neurol Neurosurg Psychiatry 66: 431-435.

5. Schrag A, Jahanshahi M, Quinn N (2000) How does parkinson's disease affect quality of life? A comparison with quality of life in the general population. Mov Disord 15: 1112-1118.

6. Duncan GW, Khoo TK, Yarnall AJ, O Brien JT, Coleman SY, et al. (2014) Health-related quality of life in early parkins disease: The impact of nonmotor symptoms. Mov Disord 29: 195-202.

7. Lawrence BJ, Gasson N, Kane R, Bucks RS, Loftus AM (2014) Activities of daily living, depression, and quality of life in parkinson's disease. PLoS One 9: e102294.

8. Litvan I, Aarsland D, Adler CH, Goldman JG, Kulisevsky J, et al. (2011) MDS task force on mild cognitive impairment in Parkinson's disease: Critical review of PD- MCl 26: 18141824.

9. Kandiah N, Zhang A, Cenina AR, Au WL, Nadkarni N, et al. (2014) Montreal cognitive assessment for the screening and prediction of cognitive decline in early parkinson's disease. Parkinsonism Relat Disord 20: 1145-1148.

10. Maira Rozenfeld O, Annelise A, Marcieli G, Artur Francisco Schumacher S, Carlos Roberto Mello R (2016) The impact of cognitive performance on quality of life in individuals with parkinson's disease. Dement Neuropsychol 10: 303-309.

11. Dirnberger G, Jahanshahi M (2013) Executive dysfunction in parkinson's disease: A review. J Neuropsychol 7: 193224.

12. Pfeiffer HCV, Løkkegaard A, Zoetmulder M, Friberg L, Werdelin $L$ (2014) Cognitive impairment in early-stage non-demented parkinson's disease patients. Acta Neurol Scand 129: 307-318.

13. Vlagsma TT, Koerts J, Fasotti L, Tucha O, Van Laar T, et al. (2016) Parkinson's patients' executive profile and goals they set for improvement: Why is cognitive rehabilitation not common practice? Neuropsychol Rehabil 26: 216-235.

14. Miller N, Noble E, Jones D, Allcock L, Burn DJ (2008) How do i sound to me? Perceived changes in communication in parkinson's disease. Clin Rehabil 22: 14-22.

15. Cahn DA, Sullivan EV, Shear PK, Pfefferbaum A, Heit G, et al. (1998) Differential contributions of cognitive and motor component processes to physical and instrumental activities of daily living in parkinson's disease. Arch Clin Neuropsychol 13: 575-583.

16. Goverover Y, Chiaravalloti N, Gaudino-Goering E, Moore N, Deluca J (2009) The relationship among performance of instrumental activities of daily living, self-report of quality of life, and self-awareness of functional status in individuals with multiple sclerosis. Rehabil Psychol 54: 60-68.

17. Klepac N, Trkulja V, Relja M, Babić T (2008) Is quality of life in non-demented parkinson's disease patients related to cognitive performance? A clinic-based cross-sectional study. Eur J Neurol 15: 128-133.

18. Guyatt G, Feeny D, Patrick D (1993) Measuring health-related quality of life. Ann Intern Med 22: 622-629.

19. Soh SE, Morris ME, McGinley JL (2011) Determinants of health-related quality of life in parkinson's disease: A systematic review. Parkinsonism Relat Disord 17: 1-9.

20. Post B, Muslimovic D, van Geloven N, Speelman JD, Schmand B, et al. (2011) Progression and prognostic fac- 
tors of motor impairment, disability and quality of life in newly diagnosed parkinson's disease. Mov Disord 26: 449-456.

21. Winter $Y$, von Cammpenhausen S, Popov G, Reese JP, Balzer-Geldsetzer M, et al. (2010) Social and clinical determinants of quality of life in Parkinson's disease in a Russian cohort study. Parkinsonism Relat Disord 16: 243-248.

22. Global Parkinson's Disease Survey (GPDS) Steering Committee (2002) Factors impacting on quality of life in parkinson's disease: Results from an international survey. Movement Disorders 17: 60-67.

23. Gómez-Esteban JC, Zarranz JJ, Lezcano E, Tijero B, Luna A, et al. (2007) Influence of motor symptoms upon the quality of life of patients with parkinson's disease. Eur Neurol 57: 161-165.

24. Altmann LJ, Troche MS (2011) High-level language production in parkinson's disease: A review. Parkinsons Dis.

25. Sapir S (2014) Multiple factors are involved in the dysarthria associated with parkinson's disease: A review with implications for clinical practice and research. J Speech Lang Hear Res 57: 1330-1343.

26. Tjaden $\mathrm{K}$ (2008) Speech and swallowing in parkinson's disease. Top Geriatr Rehabil 24: 115-126.

27. Hartelius L, Svensson P (1994) Speech and swallowing symptoms associated with parkinson's disease and multiple sclerosis: A survey. Folia Phoniatr Logop 46: 9-17.

28. Miller N, Allcock L, Jones D, Noble E, Hildreth AJ, et al (2007) Prevalence and pattern of perceived intelligibility changes in Parkinson's disease. J Neurol Neurosurg Psychiatry 78: 1188-1190.

29. Maxwell S Barnish, Simon MC Horton, Zoe R Butterfint, Allan B Clark, Rachel A Atkinson, et al. (2017) Speech and communication in parkinson's disease: A cross-sectional exploratory study in the uk. BMJ Open 7.

30. McAuliffe MJ, Baylor CR, Yorkston KM (2017) Variables associated with communicative participation in Parkinson's disease and its relationship to measures of health-related quality-of-life. Int J Speech Lang Pathol 19: 407-417.

31. Miller N (2017) Communication changes in Parkinson's disease. Pract Neurol 17: 266-274.

32. Sapir S, Ramig L, Fox C (2008) Speech and swallowing disorders in parkinson disease. Curr Opin Otolaryngol Head Neck Surg 16: 205-210.

33. Mach H, Baylor C, Pompon R, Yorkston K (2019) Third-party disability in family members of people with communication disorders associated with parkinson's disease. Top Lang Disorders 39: 71-88.

34. Baylor C, Yorkston K, Eadie T, Kim J, Chung $\mathrm{H}$, et al (2013) The communicative participation item bank (CPIB): Item bank alibration and development of a disorder-generic short form. J Speech Lang Hear Res 56: 1190-1208.

35. Eadie TL, Yorkston KM, Klasner ER, Dudgeon BJ, Deitz JC, et al. (2006) Measuring communicative participation: A review of self- report instruments in speech-language pathology. Am J Speech Lang Pathol 15: 307-320.

36. Goetz CG, Fahn S, Martinez-Martin P, Poewe W, Sampaio C, et al. (2007) Movement disorder society-sponsored revision of the unified parkinson's disease rating scale (MDS-UPDRS): Process, format, and clinimetric testing plan. Mov Disord 22: 41-47.

37. Jankovic J, McDermott M, Carter J, Gauthier S, Goetz C, et al. (1990) Variable expression of parkinson's disease: A baseline analysis of the DATATOP cohort. Neurology 40 : 1529-1534.

38. Yorkston KM, Beukelman D, Hakel M (1996) Sentence Intelligibility Test (Version 1.0). In: D Beukelman: Communication Disorders Software.

39. Tjaden K, Wilding G (2011) Effects of speaking task on intelligibility in parkinson's disease. Clin Linguist Phon 25: 155-168.

40. Sidtis D, Cameron K, Bonura L, Sidtis J J (2012) Speech intelligibility by listening in parkinson speech with and without deep brain stimulation: Task effects. Journal of Neurolinguistics 25: 121-132.

41. Goldman Eisler F (1961) The predictability of words in context and the length of pauses in speech. Journal of Communication 11: 95-99.

42. Nasreddine ZS, Phillips NA, Bédirian V, Charbonneau S, Whitehead V, et al. (2005) The montreal cognitive assessment, MoCA: A brief screening tool for mild cognitive impairment. J Am Geriatr Soc 53: 695-699.

43. Brown DS, Bernstein IH, McClintock SM, Munro Cullum C, Dewey RB, et al. (2016) Use of the montreal cognitive assessment and alzheimer's disease- 8 as cognitive screening measures in parkinson's disease. Int J Geriatr Psychiatry 31: 264-272.

44. Hoops S, Nazem S, Siderowf A D, Duda J E, Xie S X, et al. (2009) Validity of the MoCA and MMSE in the detection of $\mathrm{MCl}$ and dementia in parkinson disease. Neurology 73: 1738-1745.

45. Jenkinson C, Fitzpatrick R, Peto V, Greenhall R, Hyman N (1997) The PDQ-8: Development and validation of a short-form parkinson's disease questionnaire. Psychology \& Health 12: 805-814.

46. Jenkinson C, Fitzpatrick R, Peto V, Greenhall R, Hyman $N$ (1997) The Parkinson's disease questionnaire (PDQ-39): Development and validation of a parkinson's disease summary index score. Age and Ageing 26: 353-357.

47. Martinez Martin $P$, Jeukens Visser M, Lyons KE, Rodriguez-Blazquez C, Selai C, et al. (2011) Health-related quality-of-life scales in parkinson's disease: Critique and recommendations. Mov Disord 26: 2371-2380.

48. Hoehn MM, Yahr MD (1967) Parkinsonism: Onset, progression and mortality. Neurology 17: 427-442.

49. Borland E, Nagga K, Nilsson PM, Minthon L, Nilsson ED, et al. (2017) The montreal cognitive assessment: Normative data from a large swedish population-based cohort. J Alzheimers Dis 59: 893-901.

50. Freitas S, Simoes MR, Alves L, Santana I (2012) Montreal cognitive assessment: Influence of sociodemographic and health variables. Arch Clin Neuropsychol 27: 165-175.

51. Weir-Mayta P, Spencer KA, Eadie TL, Yorkston K, Savaglio $S$, et al. (2017) Internally versus externally cued speech in parkinson's disease and cerebellar disease. Am J Speech Lang Pathol 26: 583-595.

52. Yorkston K, Baylor C, Mach H (2017) Factors associated with communicative participation in amyotrophic lateral sclerosis. J Speech Lang Hear Res 60: 1791-1797.

53. Bunton K, Keintz CK (2008) The use of a dual-task paradigm for assessing speech intelligibility in clients with parkinson disease. J Med Speech Lang Pathol 16: 141-155.

54. Schrag A, Jahanshahi M, Quinn N (2000) What contributes to quality of life in patients with Parkinson's disease. J Neurol Neurosurg Psychiatry 69: 308-312. 